\title{
Article \\ Bayesian Analysis of Dynamic Cumulative Residual Entropy for Lindley Distribution
}

\author{
Abdullah M. Almarashi ${ }^{1}$, Ali Algarni ${ }^{1}$, Amal S. Hassan ${ }^{2}$, Ahmed N. Zaky ${ }^{3}$ (D) and Mohammed Elgarhy ${ }^{4, *(D)}$ \\ 1 Statistics Department, Faculty of Science, King Abdulaziz University, Jeddah 21551, Saudi Arabia; \\ aalmarashi@kau.edu.sa (A.M.A.); ahalgarni@kau.edu.sa (A.A.) \\ 2 Faculty of Graduate Studies for Statistical Research, Cairo University, Giza 12613, Egypt; \\ amal52_soliman@cu.edu.eg \\ Institute of National Planning, Cairo 11765, Egypt; ahmed.nasser@inp.edu.eg \\ 4 The Higher Institute of Commercial Sciences, Al Mahalla Al Kubra, Algarbia 31951, Egypt \\ * Correspondence: M_elgarhy85@sva.edu.eg
}

check for updates

Citation: Almarashi, A.M.; Algarni,

A.; Hassan, A.S.; Zaky, A.N.; Elgarhy,

M. Bayesian Analysis of Dynamic Cumulative Residual Entropy for Lindley Distribution. Entropy 2021, 23, 1256. https://doi.org/10.3390/ e23101256

Academic Editor: Boris Ryabko

Received: 19 August 2021

Accepted: 23 September 2021

Published: 27 September 2021

Publisher's Note: MDPI stays neutral with regard to jurisdictional claims in published maps and institutional affiliations.

Copyright: (c) 2021 by the authors. Licensee MDPI, Basel, Switzerland. This article is an open access article distributed under the terms and conditions of the Creative Commons Attribution (CC BY) license (https:// creativecommons.org/licenses/by/ $4.0 /)$.
Abstract: Dynamic cumulative residual (DCR) entropy is a valuable randomness metric that may be used in survival analysis. The Bayesian estimator of the DCR Rényi entropy (DCRRéE) for the Lindley distribution using the gamma prior is discussed in this article. Using a number of selective loss functions, the Bayesian estimator and the Bayesian credible interval are calculated. In order to compare the theoretical results, a Monte Carlo simulation experiment is proposed. Generally, we note that for a small true value of the DCRRéE, the Bayesian estimates under the linear exponential loss function are favorable compared to the others based on this simulation study. Furthermore, for large true values of the DCRRéE, the Bayesian estimate under the precautionary loss function is more suitable than the others. The Bayesian estimates of the DCRRéE work well when increasing the sample size. Real-world data is evaluated for further clarification, allowing the theoretical results to be validated.

Keywords: Rényi entropy; Lindley distribution; Bayesian estimators; squared error loss function

\section{Introduction}

Reference [1] introduced the idea of the Rényi entropy as a measure of randomness for Y. The Rényi entropy can be used to estimate the uncertainty in a random observation. In the study of quantum systems, quantum communication protocols, and quantum correlations [2,3], it has been extensively utilized. The probability density function (PDF) $\mathrm{g}($.$) and the distribution function (CDF) \mathrm{G}($.$) of the Rényi entropy with the order \beta$ is given by

$$
\Xi(\beta)=(1-\beta)^{-1} \log \left(\int_{-\infty}^{\infty} g^{\beta}(y) d y\right), \beta>0, \beta \neq 1 .
$$

In recent times, several authors studied the statistical inferences for the entropy measures using different distributions and sampling schemes (for example, [4-12]).

Alternative measurements of uncertainty for probability distributions in recent times are of interest to many authors, especially in reliability and survival analysis studies. Therefore, the cumulative residual entropy and its dynamic version have been proposed, respectively, in $[13,14]$. The DCRRéE is defined as follows:

$$
\gamma_{R}(\beta)=(1-\beta)^{-1} \log \left(\int_{t}^{\infty} \frac{\bar{G}^{\beta}(y)}{\bar{G}^{\beta}(t)} d y\right), \beta>0, \beta \neq 1 .
$$

where $\bar{G}(t)=1-G(t)$ is the survival function (SF), and for $t=0$, the DCRRéE leads to the cumulative residual Rényi entropy. In the literature, few works have been regarded 
for the inferential procedures of DCR entropy for lifetime distributions. Properties of the DCR entropy from the order statistics were presented in [15]. The cumulative residual and past inaccuracy have been proposed in [16] as extensions of the cumulative entropies for the truncated random variables. The Bayesian estimators of the DCR entropy of the Pareto model using different sampling schemes have been studied in [17-19]. The Bayesian inference of the DCR entropy for the Pareto II distribution was given in [20]. The Bayesian and non-Bayesian estimators of the DCR entropy for the Lomax distribution were provided in [21].

Reference [22] was the first to use the Lindley distribution to evaluate failure time data, particularly in reliability modeling. It is also a good alternative to the exponential distribution since it combines the exponential and gamma distributions. Hazard rates might be increasing, decreasing, uni-modal, or bathtub-shaped, resulting in the modeling of multiple lifetime data. The PDF of the Lindley distribution is

$$
g(y ; \theta)=\frac{\theta^{2}}{\theta+1}(1+y) e^{-\theta y} ; \quad y, \theta>0 .
$$

The CDF and the SF of the Lindley distribution are given by

$$
G(y ; \theta)=1-e^{-\theta y}\left[1+\frac{\theta y}{\theta+1}\right] ; \quad y, \theta>0,
$$

and

$$
\bar{G}(y ; \theta)=e^{-\theta y}\left[1+\frac{\theta y}{\theta+1}\right] ; \quad y, \theta>0 .
$$

The authors of $[23,24]$ handled the properties and the inferential procedure for the Lindley distribution. As a result, numerous writers have utilized the Lindley distribution to predict lifetime data under intended circumstances; see [25-30] and the references listed therein.

To generate random numbers from the Lindley distribution, we may use the fact that the distribution, as given in Equation (3), is a mixture of exponential $(\theta)$ and gamma $(2, \theta)$, with mixing proportions $(\theta / 1+\theta)$ and $(1 / 1+\theta)$, respectively. For generating a random sample of size $n$, we have the following simulation algorithm:

(i) Generate $\boldsymbol{U}_{i}$ from uniform $(0,1), i=1,2, \ldots, n$.

(ii) Generate $Q_{i}$ from exponential $(\theta), i=1,2, \ldots, n$.

(iii) Generate $V_{i}$ from gamma $(2, \theta), i=1,2, \ldots, n$.

(iv) If $U_{i} \leq \boldsymbol{\theta} / \mathbf{1}+\boldsymbol{\theta}$ then set $X_{i}=Q_{i}$, otherwise, set $X_{i}=V_{i}$.

Since the last decade, the Lindley distribution has attracted the attention of researchers for its use in several fields as well as for modeling lifetime data. Herein, we intend to discuss the Bayesian inference of the DCRRéE for the Lindley model. The Bayesian estimators and the Bayesian credible intervals of the DCRRéE under the gamma prior are derived. The proposed estimators are obtained via the squared error (SE), linear exponential (LINEx), and precautionary (PR) loss functions. The Markov Chain Monte Carlo (MCMoC) simulation is utilized because the DCRRéE's Bayesian estimator is complicated. A real data analysis is given for illustration. We outline the paper as follows: Section 2 gives the formula for the DCRRéE of the Lindley distribution; Section 3 offers the DCRRéE's Bayesian estimator of the Lindley distribution under the specific loss functions; a description of MCMoC is provided in Section 4; and in Section 5, a real-world data application is shown. Using the findings of our numerical investigations, we came to certain conclusions. 


\section{Expression of the DCRRéE for the Lindley Distribution}

This section presents the formula of the DCRRéE for the Lindley distribution. The DCRRéE of the Lindley distribution is obtained by substituting Equation (5) into Equation (2) as follows:

$$
\gamma_{R}(\beta)=\frac{1}{(1-\beta)} \log \left(\frac{1}{\bar{G}^{\beta}(t)} \int_{t}^{\infty} e^{-\theta \beta y}\left(1+\frac{\theta y}{\theta+1}\right)^{\beta}\right)=\frac{1}{(1-\beta)} \log \left(\frac{1}{\bar{G}^{\beta}(t)} I\right),
$$

where $I=\int_{t}^{\infty} e^{-\theta \beta y}\left(1+\frac{\theta y}{\theta+1}\right)^{\beta} d y$. To obtain $I$, we use the transformation $x=1+\frac{\theta y}{\theta+1}$, then we have

$$
I=\left(1+\frac{1}{\theta}\right) \int_{1+\frac{\theta t}{\theta+1}}^{\infty} e^{-\beta(1+\theta)(x-1)} x^{\beta} d x=\left(1+\frac{1}{\theta}\right) e^{\beta(1+\theta)} \int_{1+\frac{\theta t}{\theta+1}}^{\infty} x^{\beta} e^{-\beta(1+\theta) x} d x .
$$

Let $z=\beta(1+\theta) x$, and then Equation (7) can be expressed as

$$
I=\left(1+\frac{1}{\theta}\right) \frac{e^{\beta(1+\theta)}}{[\beta(1+\theta)]^{\beta+1}} \int_{\beta(1+\theta+\theta t)}^{\infty} z^{\beta} e^{-z} d z=\frac{e^{\beta \mathrm{A}(\theta)} \Gamma(\beta+1, \beta \mathrm{A}(\theta))}{\theta \beta[\mathrm{A}(\theta)]^{\beta}},
$$

where $\Gamma($.$) stands for an incomplete gamma function and \mathrm{A}(\theta)=1+\theta+\theta t$. By substituting Equation (8) into Equation (6), the DCRRéE for the Lindley distribution is expressed as follows

$$
\gamma_{R}(\beta)=\frac{1}{1-\beta} \log \left(\frac{e^{\beta \mathrm{A}(\theta)} \Gamma(\beta+1, \beta \mathrm{A}(\theta))}{\theta \beta[\mathrm{A}(\theta)]^{\beta}}\right) .
$$

The DCRRéE requires this phrase for the Lindley distribution.

\section{The Bayesian Estimation}

Herein, the Bayesian estimator of the DCRRéE for the Lindley distribution is obtained using the gamma prior. The Bayesian estimator is derived under the selected loss functions, and the Bayesian credible intervals are computed.

A random sample of size $n$ taken from the PDF (3) and the CDF (4) can be used if $\theta$ is unknown. Then, the likelihood function of the Lindley distribution given the sample $\underline{y}=\left(y_{1}, \ldots, y_{n}\right)$, is given by

$$
l(\theta \mid \underline{y})=\frac{\theta^{2 n}}{(\theta+1)^{n}} e^{-\theta \sum_{i=1}^{n} y_{i}} \prod_{i=1}^{n}\left(1+y_{i}\right) .
$$

Let us assume that the prior of $\theta$ has a gamma distribution with the parameters $(a, b)$ with the following PDF

$$
\pi(\theta)=\frac{a^{b}}{\Gamma(b)} \theta^{b-1} e^{-\theta a}, \quad a, b>0 .
$$

This is how the posterior PDF of $\theta$ given the data can be expressed as

$$
\pi(\theta \mid \underline{y})=k \frac{a^{b} \theta^{2 n+b-1}}{(\theta+1)^{n} \Gamma(b)} \exp \left\{\sum_{i=1}^{n} \ln \left(1+y_{i}\right)-\theta \sum_{i=1}^{n} y_{i}-a \theta\right\}
$$

where

$$
k^{-1}=\int_{0}^{\infty} \frac{a^{b} \theta^{2 n+b-1}}{(\theta+1)^{n} \Gamma(b)} \exp \left\{\sum_{i=1}^{n} \ln \left(1+y_{i}\right)-\theta \sum_{i=1}^{n} y_{i}-a \theta\right\} d \theta
$$


The Bayes estimator of $\gamma_{R}(\beta)$ under the SE loss function, denoted by $\hat{\gamma}_{S E}(\beta)$, is obtained as follows:

$$
\begin{aligned}
\hat{\gamma}_{S E}(\beta) & =\int_{0}^{\infty} \gamma_{R}(\beta) \pi(\theta \mid \underline{y}) d \theta \\
& =\frac{k}{1-\beta} \int_{0}^{\infty} \log \left(\frac{e^{\beta \mathrm{A}(\theta)} \Gamma(\beta+1, \beta \mathrm{A}(\theta))}{\theta \beta[\mathrm{A}(\theta)]^{\beta}}\right) \frac{a^{b} \theta^{2 n+b-1}}{(\theta+1)^{n} \Gamma(b)} \exp \left\{\sum_{i=1}^{n} \ln \left(1+y_{i}\right)-\theta \sum_{i=1}^{n} y_{i}-a \theta\right\} d \theta .
\end{aligned}
$$

Based on the LINEx loss function, the Bayes estimator of $\gamma_{R}(\beta)$ says $\hat{\gamma}_{\text {LINEx }}(\beta)$ is given by

$$
\begin{aligned}
\hat{\gamma}_{\text {LINEx }}(\beta) & =\frac{-1}{v} \ln \left[\int_{0}^{\infty} e^{-v} \gamma_{R}(\beta) \pi(\theta \mid \underline{y}) d \theta\right], v \neq 0, \\
= & \frac{-1}{v} \ln \left[k \int_{0}^{\infty}\left(\frac{e^{\beta \mathrm{A}(\theta)} \Gamma(\beta+1, \beta \mathrm{A}(\theta))}{\theta \beta[\mathrm{A}(\theta)]^{\beta}}\right)^{\frac{-v}{\beta-1}} \frac{a^{b} \theta^{2 n+b-1}}{(\theta+1)^{n} \Gamma(b)} \exp \left\{\sum_{i=1}^{n} \ln \left(1+y_{i}\right)-\theta \sum_{i=1}^{n} y_{i}-a \theta\right\} d \theta\right] .
\end{aligned}
$$

Using the PR loss function, the Bayes estimator of $\gamma_{R}(\beta)$ says $\hat{\gamma}_{P R}(\beta)$ is given by

$$
\begin{aligned}
\hat{\gamma}_{P R}(\beta) & =\left[\int_{0}^{\infty}\left(\gamma_{R}(\beta)\right)^{2} \pi(\theta \mid \underline{y}) d \theta\right]^{0.5} \\
& =\left[\frac{k}{1-\beta} \int_{0}^{\infty}\left\{\log \left(\frac{e^{\beta \mathrm{A}(\theta)} \Gamma(\beta+1, \beta \mathrm{A}(\theta))}{\theta \beta[\mathrm{A}(\theta)]^{\beta}}\right)\right\}^{2} \frac{a^{b} \theta^{2 n+b-1}}{(\theta+1)^{n} \Gamma(b)} \exp \left\{\sum_{i=1}^{n} \ln \left(1+y_{i}\right)-\theta \sum_{i=1}^{n} y_{i}-a \theta\right\} d \theta\right]^{0.5} .
\end{aligned}
$$

As previously stated, the analytical solution to Integrations (11-13) is extremely difficult to acquire due to complex mathematical forms. To approximate these integrations, the MCMoC technique is used. Furthermore, using the method described in [31], we obtain the Bayesian credible intervals of $\gamma_{R}(\beta)$. A credible interval is the Bayesian equivalent of a confidence interval. The upper $(\mathrm{U})$ and lower $(\mathrm{L})$ credible limits are the $\mathrm{U}$ and $\mathrm{L}$ endpoints of a credible interval, respectively.

The probability that a credible interval will contain the unknown parameter $\theta$ is called the "confidence coefficient". If we suppose the $\mathrm{L}$ and $\mathrm{U}$ credible limits, respectively, for the parameter $\theta$, then $P(L<\theta<U)=1-\eta$, where $(1-\eta) 100 \frac{0}{0}$ is the confidence coefficient.

\section{Numerical Illustrations and Results}

For the Lindley distribution at $\beta=0.5$, a numerical analysis is conducted in this part to examine the performance of the Bayesian estimates of $\gamma_{R}(\beta)$. In Bayesian literature, the Metropolis-Hastings ( $\mathrm{MH}$ ) algorithm (see [32]) is one of the most well-known subclasses of the MCMoC technique for simulating deviations from the posterior density and producing good approximation results. MCMoC simulations are run for selected sample sizes and loss functions. R 4.1.1 will be used to run the $\mathrm{MH}$ algorithm.

The MCMoC method is used to generate samples from the posterior distributions and then to compute the DCRRéE's Bayesian estimators under the intended loss functions. $\mathrm{MCMoC}$ schemes come in a wide range of options. Gibbs sampling and the more general Metropolis-within-Gibbs samplers are a significant subclass of the MCMoC methods.

To pull samples from the posterior density functions and then compute the Bayesian estimators, we use the following MCMoC technique, see Algorithm 1.

The hyper-parameters of the gamma distribution are specified as $a=2$ and $b=1$. Choose $\mathrm{v}=(-1,1)$ for the LINEx loss function, which represents underestimation and overestimation, respectively. Using a sample size of 5,000, $n=30,50,70$, and 100 are generated from the Lindley model. The true values of the parameter values are chosen as $\theta=(0.8,1.5,2)$. The actual value of the DCRRéE measure is elected as $\gamma_{R}(\beta)=2.433289127$, 1.025114899. 0.38237199 where $t=0.5$, and $\gamma_{R}(\beta)=2.31065,0.90832$. 0.27434 where $t=1.5$. Measures including the RABs and the ERs of the Bayes estimates (Bes) of the DCRRéE, along with the width (WD) of the Bayesian credible interval, are computed. 


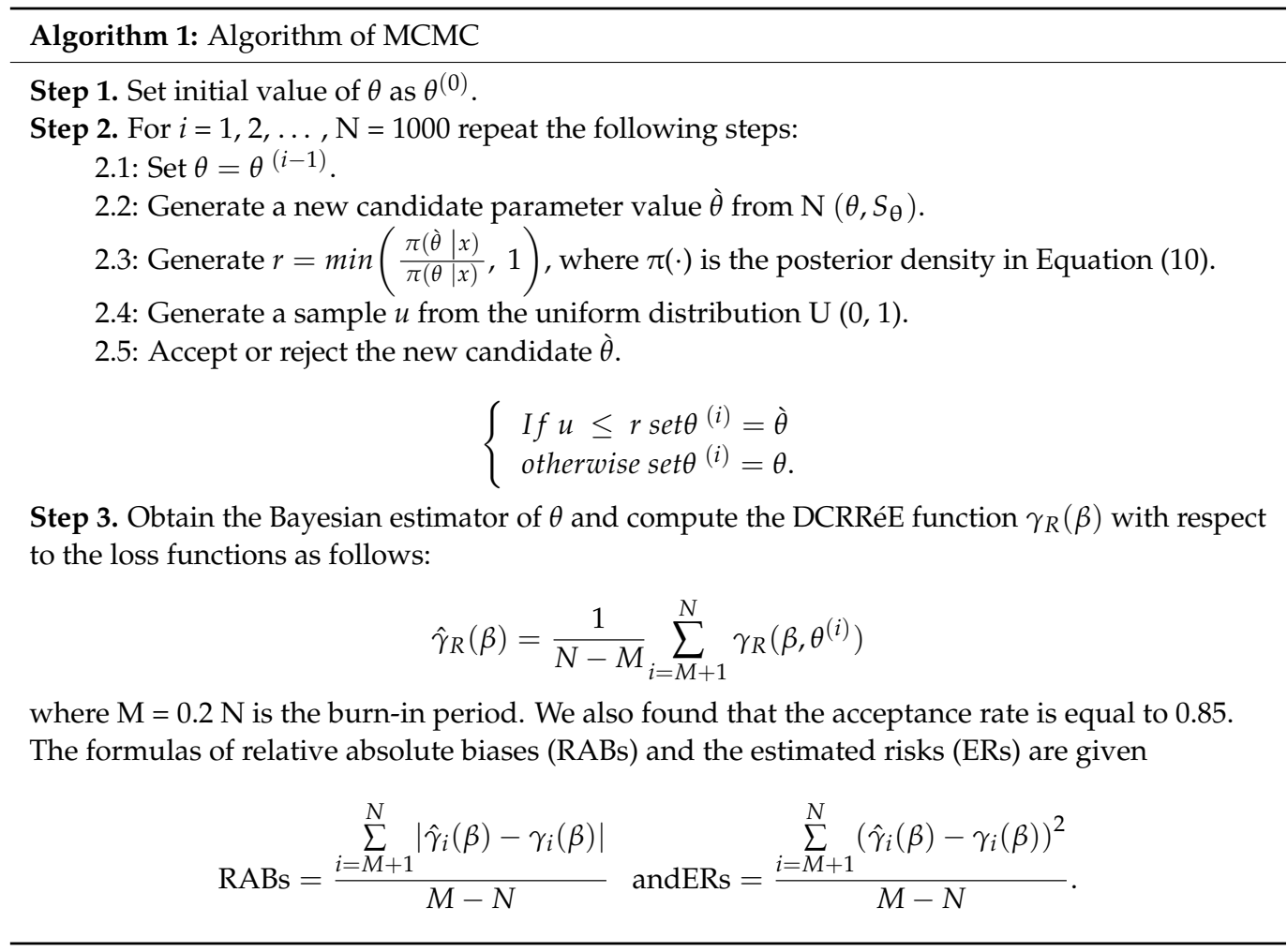

\subsection{Numerical Results}

The results of this study are presented in Tables 1-3 for the DCRRéE estimates at $t=0.5$, and Tables 4-6 give the simulation results for the DCRRéE estimates at $t=1.5$. Figures 1-4 also provide the numerical results. Accordingly, we may draw the following conclusions about the DCRRéE estimates.

- $\quad$ As the $\theta$ value grows, the DCRRéE estimates appear smaller for a similar value of $t$.

- The DCRRéE estimates decrease with an increasing value of $t$ for a similar value of $\theta$. At $t=0.5$, the following notes can be recorded:

- The estimated risk of $\hat{\gamma}_{\text {LINEx }}(\beta)$ at $\mathrm{v}=-1$ picks the lowest values for $n=50$ and 70 while the estimated risk of $\hat{\gamma}_{\text {LINEx }}(\beta)$ at $\mathrm{v}=1$ picks the lowest values at $n=100$. In addition, the width of the credible interval for $\hat{\gamma}_{\operatorname{LINEx}}(\beta)$ at $\mathrm{v}=-1$ takes the lowest values for $n=100$ (see Table 1 ).

- The estimated risk of $\hat{\gamma}_{P R}(\beta)$ has the lowest values for all $n$ values, and the width of the credible interval for $\hat{\gamma}_{P R}(\beta)$ picks the lowest values for all values of $n$ except $n=70$ (see Table 2).

- At actual value $\gamma_{R}(\beta)=0.38237199(\theta=2.0)$, the estimated risk of $\hat{\gamma}_{\text {LINEx }}(\beta)$ at $\mathrm{v}=1$ for all $n$ values except at $n=100$ has the lowest values. Moreover, the width of the credible interval for $\hat{\gamma}_{\text {LINEx }}(\beta)$ at $\mathrm{v}=1$ obtains the lowest value at $n=70$ (see Table 3).

- We can see from Figure 1 that the estimated risk for $\hat{\gamma}_{P R}(\beta)$ at the true value $\gamma_{R}(\beta)>1$ for $n=30$ has the lowest values when compared to the other estimates, except at the true value of $\gamma_{R}(\beta)=0.38237$.

- $\quad$ Figure 2 indicates that the estimated risks of $\hat{\gamma}_{\text {LINEx }}(\beta)$ at $\mathrm{v}=1$ have the lowest value at $\gamma_{R}(\beta)=2.43328$ when compared to the other estimates for $n=100$. 
Table 1. Measures of Accuracy for DCRRéE at $\theta=0.8, t=0.5$, and $\gamma_{R}(\beta)=2.433289$.

\begin{tabular}{|c|c|c|c|c|c|c|c|c|c|c|c|c|c|c|c|c|}
\hline \multirow{2}{*}{$n$} & \multicolumn{4}{|c|}{ SE } & \multicolumn{4}{|c|}{ LINEx $(v=1)$} & \multicolumn{4}{|c|}{ LINEx $(v=-1)$} & \multicolumn{4}{|c|}{ PR } \\
\hline & BE & RAB & ER & WD & BE & RAB & ER & WD & BE & RAB & ER & WD & BE & RAB & ER & WD \\
\hline 30 & 2.42926 & 0.00166 & 0.06132 & 0.94796 & 2.42550 & 0.00320 & 0.06121 & 0.94596 & 2.43302 & 0.00011 & 0.06146 & 0.95129 & 2.42450 & 0.00361 & 0.06088 & 0.94389 \\
\hline 70 & 2.41950 & 0.00567 & 0.03941 & 0.75725 & 2.41658 & 0.00687 & 0.03951 & 0.75845 & 2.42242 & 0.00447 & 0.03934 & 0.75966 & 2.41587 & 0.00716 & 0.03939 & 0.75794 \\
\hline 100 & 2.43023 & 0.00126 & 0.02889 & 0.66839 & 2.42763 & 0.00233 & 0.02894 & 0.66955 & 2.43284 & 0.00018 & 0.02885 & 0.66541 & 2.42699 & 0.00259 & 0.02887 & 0.66919 \\
\hline
\end{tabular}

Table 2. Measures of Accuracy for DCRRéE at $\theta=1.5, t=0.5$, and $\gamma_{R}(\beta)=1.02511$.

\begin{tabular}{|c|c|c|c|c|c|c|c|c|c|c|c|c|c|c|c|c|}
\hline \multirow{2}{*}{$n$} & \multicolumn{4}{|c|}{ SE } & \multicolumn{4}{|c|}{ LINEx $(v=1)$} & \multicolumn{4}{|c|}{ LINEx $(v=-1)$} & \multicolumn{4}{|c|}{ PR } \\
\hline & BE & RAB & ER & WD & BE & RAB & ER & WD & BE & RAB & ER & WD & BE & RAB & ER & WD \\
\hline 30 & 1.02767 & 0.00250 & 0.02292 & 0.59123 & 1.02535 & 0.00023 & 0.022793 & 0.59016 & 1.03000 & 0.00477 & 0.02306 & 0.59175 & 1.02610 & 0.00096 & 0.022787 & 0.59015 \\
\hline 50 & 1.03075 & 0.00550 & 0.02128 & 0.56636 & 1.02856 & 0.00336 & 0.02123 & 0.56555 & 1.03295 & 0.00764 & 0.02135 & 0.56662 & 1.02928 & 0.00406 & 0.02121 & 0.56545 \\
\hline 70 & 1.01934 & 0.00563 & 0.02024 & 0.54244 & 1.01716 & 0.00776 & 0.02025 & 0.53970 & 1.02153 & 0.00350 & 0.02025 & 0.54470 & 1.01788 & 0.00706 & 0.02021 & 0.54031 \\
\hline 100 & 1.02838 & 0.00319 & 0.01682 & 0.48992 & 1.02636 & 0.00121 & 0.01676 & 0.49011 & 1.03041 & 0.00516 & 0.01689 & 0.49149 & 1.02702 & 0.00186 & 0.01675 & 0.48942 \\
\hline
\end{tabular}

Table 3. Measures of Accuracy for DCRRéE for $\theta=2.0, t=0.5$, and $\gamma_{R}(\beta)=0.38237$.

\begin{tabular}{|c|c|c|c|c|c|c|c|c|c|c|c|c|c|c|c|c|}
\hline \multirow{2}{*}{$n$} & \multicolumn{4}{|c|}{ SE } & \multicolumn{4}{|c|}{ LINEx $(v=1)$} & \multicolumn{4}{|c|}{ LINEx $(v=-1)$} & \multicolumn{4}{|c|}{ PR } \\
\hline & BE & RAB & ER & WD & BE & RAB & ER & WD & $\mathrm{BE}$ & RAB & ER & WD & $\mathrm{BE}$ & RAB & ER & WD \\
\hline 30 & 0.39342 & 0.02888 & 0.01409 & 0.45858 & 0.39158 & 0.02408 & 0.01403 & 0.45810 & 0.39525 & 0.03368 & 0.01415 & 0.45534 & 0.39249 & 0.02646 & 0.01404 & 0.45710 \\
\hline 50 & 0.38557 & 0.00835 & 0.01334 & 0.43146 & 0.38381 & 0.00377 & 0.01330 & 0.43208 & 0.38732 & 0.01294 & 0.01339 & 0.43187 & 0.38468 & 0.00604 & 0.01331 & 0.43033 \\
\hline 70 & 0.38513 & 0.00721 & 0.01254 & 0.42933 & 0.38347 & 0.00287 & 0.01252 & 0.42705 & 0.38679 & 0.01156 & 0.01257 & 0.42750 & 0.38429 & 0.00503 & 0.01257 & 0.42820 \\
\hline 100 & 0.38601 & 0.00952 & 0.01141 & 0.41235 & 0.38437 & 0.00522 & 0.01140 & 0.41399 & 0.38766 & 0.01383 & 0.01142 & 0.41399 & 0.38519 & 0.00736 & 0.01139 & 0.41346 \\
\hline
\end{tabular}


Table 4. Measures of Accuracy for DCRRéE for $\theta=0.8, t=1.5$, and $\gamma_{R}(\beta)=2.31065$

\begin{tabular}{|c|c|c|c|c|c|c|c|c|c|c|c|c|c|c|c|c|}
\hline \multirow{2}{*}{$n$} & \multicolumn{4}{|c|}{ SE } & \multicolumn{4}{|c|}{ LINEx (v = 1) } & \multicolumn{4}{|c|}{ LINEx $(v=-1)$} & \multicolumn{4}{|c|}{ PR } \\
\hline & BE & RAB & ER & WD & BE & $\mathrm{RAB}$ & ER & WD & BE & RAB & ER & WD & BE & RAB & ER & WD \\
\hline 30 & 2.34019 & 0.01279 & 0.05839 & 0.92243 & 2.33632 & 0.01111 & 0.05811 & 0.92058 & 2.34407 & 0.01446 & 0.05871 & 0.91961 & 2.33524 & 0.01064 & 0.05774 & 0.91789 \\
\hline 70 & 2.29300 & 0.00764 & 0.03709 & 0.74947 & 2.29013 & 0.00888 & 0.03725 & 0.75022 & 2.29586 & 0.00640 & 0.03695 & 0.74925 & 2.28945 & 0.00918 & 0.03717 & 0.74970 \\
\hline 100 & 2.28540 & 0.01093 & 0.03098 & 0.65564 & 2.28284 & 0.01204 & 0.03112 & 0.65423 & 2.28797 & 0.00982 & 0.03087 & 0.65897 & 2.28222 & 0.01230 & 0.03106 & 0.65275 \\
\hline
\end{tabular}

Table 5. Measures of Accuracy for DCRRéE for $\theta=1.5, t=1.5$, and $\gamma_{R}(\beta)=0.90832$.

\begin{tabular}{|c|c|c|c|c|c|c|c|c|c|c|c|c|c|c|c|c|}
\hline \multirow{2}{*}{$n$} & \multicolumn{4}{|c|}{ SE } & \multicolumn{4}{|c|}{ LINEx $(v=1)$} & \multicolumn{4}{|c|}{ LINEx $(v=-1)$} & \multicolumn{4}{|c|}{ PR } \\
\hline & BE & RAB & ER & WD & BE & RAB & ER & WD & $\mathrm{BE}$ & RAB & ER & WD & BE & RAB & ER & WD \\
\hline 30 & 0.91976 & 0.01260 & 0.02292 & 0.56958 & 0.91744 & 0.01005 & 0.022792 & 0.56538 & 0.92208 & 0.01515 & 0.02306 & 0.57188 & 0.91819 & 0.01087 & 0.022788 & 0.56644 \\
\hline 70 & 0.89959 & 0.00961 & 0.02005 & 0.54054 & 0.89749 & 0.01192 & 0.02081 & 0.53003 & 0.90169 & 0.00730 & 0.02011 & 0.53211 & 0.89820 & 0.01114 & 0.02076 & 0.54979 \\
\hline 100 & 0.91480 & 0.00714 & 0.01771 & 0.51330 & 0.91285 & 0.00499 & 0.01765 & 0.51441 & 0.91675 & 0.00928 & 0.01779 & 0.51429 & 0.91349 & 0.00569 & 0.01764 & 0.51315 \\
\hline
\end{tabular}

Table 6. Measures of Accuracy for DCRRéE at $\theta=2.0, t=1.5$, and $\gamma_{R}(\beta)=0.27434$.

\begin{tabular}{|c|c|c|c|c|c|c|c|c|c|c|c|c|c|c|c|c|}
\hline \multirow{2}{*}{$n$} & \multicolumn{4}{|c|}{ SE } & \multicolumn{4}{|c|}{ LINEx (v = 1) } & \multicolumn{4}{|c|}{ LINEx $(v=-1)$} & \multicolumn{4}{|c|}{ PR } \\
\hline & BE & RAB & ER & WD & BE & RAB & ER & WD & BE & RAB & ER & WD & BE & RAB & ER & WD \\
\hline 30 & 0.28367 & 0.03401 & 0.01306 & 0.43538 & 0.28192 & 0.02763 & 0.01296 & 0.43471 & 0.28542 & 0.04039 & 0.01318 & 0.43649 & 0.28278 & 0.03077 & 0.01300 & 0.43461 \\
\hline 50 & 0.27769 & 0.01221 & 0.01285 & 0.43401 & 0.27596 & 0.00591 & 0.012817 & 0.43286 & 0.27942 & 0.01853 & 0.01290 & 0.43440 & 0.27682 & 0.00903 & 0.01282 & 0.43410 \\
\hline 70 & 0.28249 & 0.02972 & 0.01277 & 0.43031 & 0.28072 & 0.02326 & 0.01272 & 0.43063 & 0.28427 & 0.03620 & 0.01283 & 0.42760 & 0.28160 & 0.02646 & 0.01273 & 0.43075 \\
\hline 100 & 0.28390 & 0.03486 & 0.01145 & 0.41154 & 0.28225 & 0.02883 & 0.01140 & 0.40894 & 0.28556 & 0.04089 & 0.01150 & 0.41205 & 0.28307 & 0.03182 & 0.01141 & 0.41037 \\
\hline
\end{tabular}




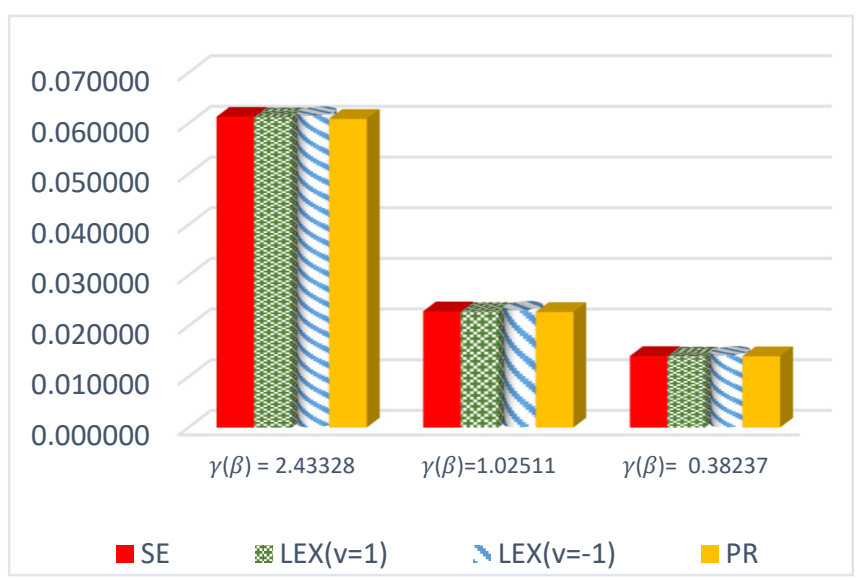

Figure 1. ER of DCRRéE estimates under proposed loss functions at $n=30$ and $t=0.5$.

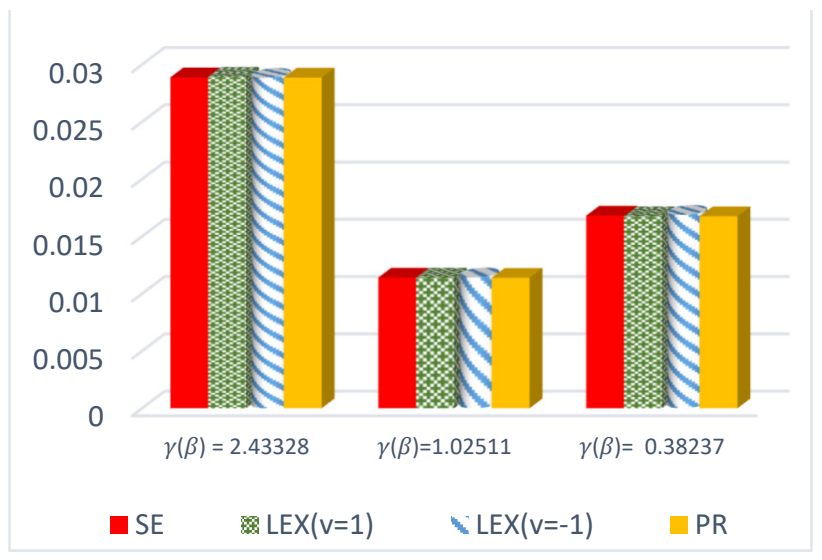

Figure 2. ER of DCRRéE estimates under proposed loss functions at $n=100$ and $t=0.5$.

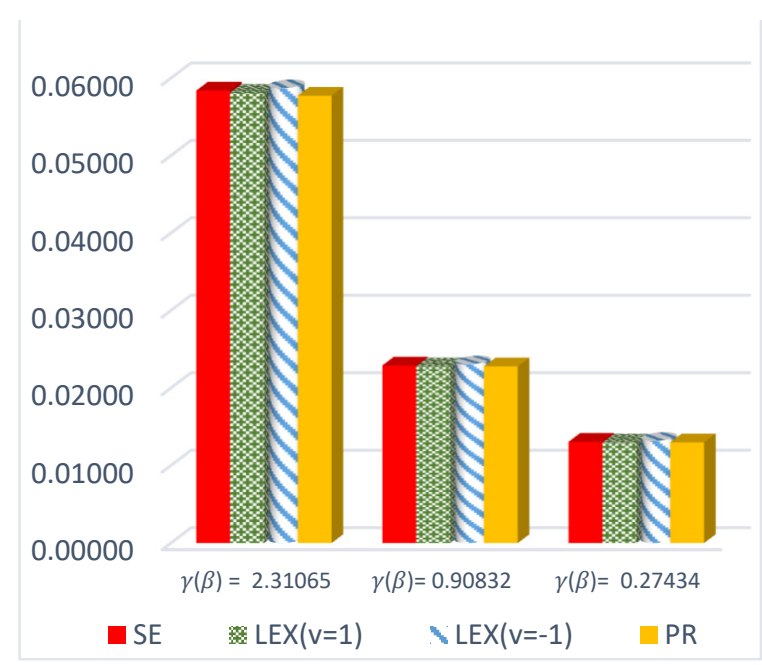

Figure 3. ER of DCRRéE estimates under proposed loss functions at $n=30$ and $t=1.5$. 


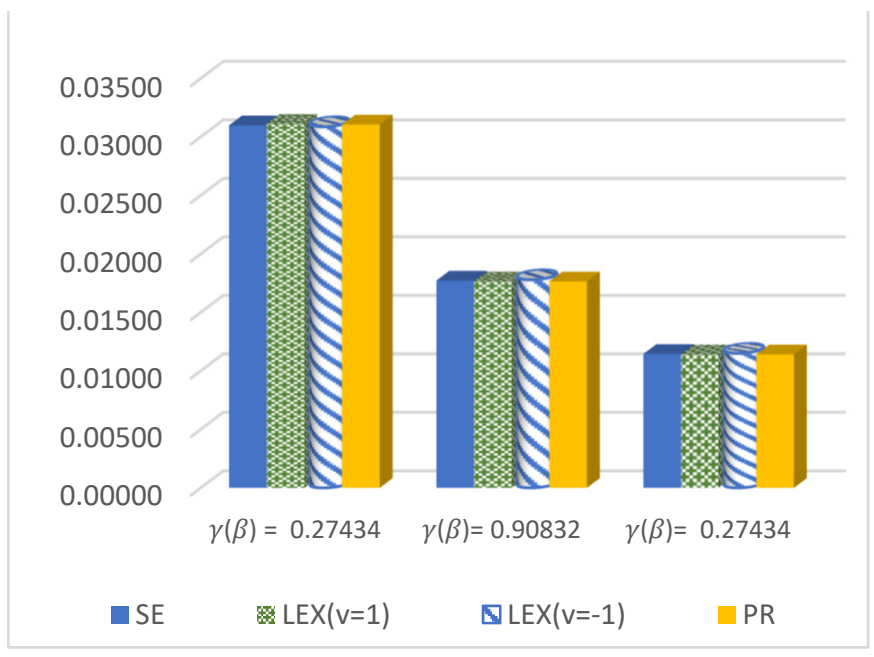

Figure 4. ER of DCRRéE estimates under proposed loss functions at $n=100$ and $t=1.5$.

The following are the notes that may be found at $t=1.5$ :

- $\quad$ The estimated risk of $\hat{\gamma}_{\text {LINEx }}(\beta)$ at $\mathrm{v}=-1$ obtains the lowest values at $n=70$ and 100 while the estimated risk of $\hat{\gamma}_{P R}(\beta)$ has the lowest values for $n=30$ and 50. The width of the Bayesian credible interval for $\hat{\gamma}_{\text {LINEx }}(\beta)$ at $\mathrm{v}=-1$ is the smallest in comparison with other estimates for $n=50$ and 70 (see Table 4 ).

- At $n=30$ and 100, the estimated risk of $\hat{\gamma}_{P R}(\beta)$ has the lowest values, while the estimated risk of $\hat{\gamma}_{S E}(\beta)$ has the lowest values at $n=50$ and 70. The width of the Bayesian credible interval for $\hat{\gamma}_{\text {LINEx }}(\beta)$ at $\mathrm{v}=1$ is the shortest compared to the others via the SE and PR loss functions, except at $n=100$ (see Table 5).

- We can see from Figure 3 that the estimated risk of $\hat{\gamma}_{P R}(\beta)$, at $n=30$ holds the lowest values for all actual values of $\gamma_{R}(\beta)$,, except at $\gamma_{R}(\beta)=0.27434$.

- For a large sample size $(n=100)$, the estimated risks for $\hat{\gamma}_{\text {LINEx }}(\beta)$ at $\mathrm{v}=1$ obtain the lowest value at actual value of $\gamma_{R}(\beta)=0.27434,0.90832$, as shown in Figure 4 .

- We conclude from Table 6 that the estimated risks of $\hat{\gamma}_{\text {LINEx }}(\beta)$ at $\mathrm{v}=1$ provide the lowest values for all values of $n$. Moreover, the width of the Bayesian credible intervals for $\hat{\gamma}_{\text {LINEx }}(\beta)$ at $\mathrm{v}=1$ takes the lowest values with respect to all possible values of $n$, except at $n=30$ and 70 .

- $\quad$ Figures 5-7 represent trace plots, histograms, and convergences for $\gamma_{R}(\beta)$ estimates using the $\mathrm{MH}$ algorithm.

\subsection{Application}

Here, we demonstrate the technique described in the preceding section by using an actual data set that represents the waiting times (in minutes) before receiving service for 100 bank customers. Reference [23] discussed the detailed statistics that showed the data fitted the Lindley distribution. Figures 8 and 9 provide plots of fitted PDF and CDF for the data under consideration. The Bayes estimates of the DCRRéE at $t=0.5$ and 1.5 at the intended loss functions are reported in Table 7. 
Plot of DCRR SE

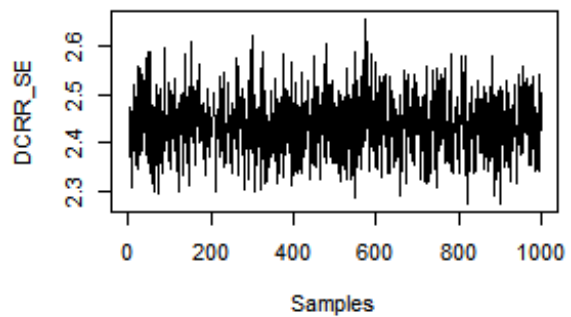

Plot of DCRR_LINEx at $v=-1$

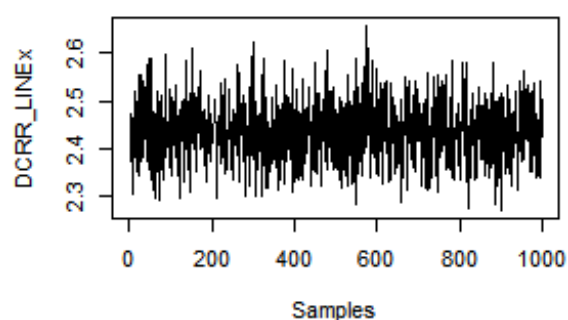

Plot of DCRR_LINEx at $v=1$

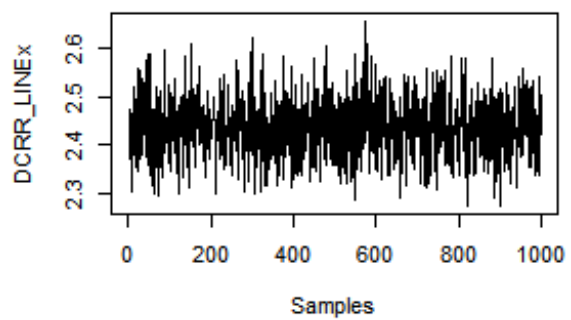

Plot of DCRR_PR

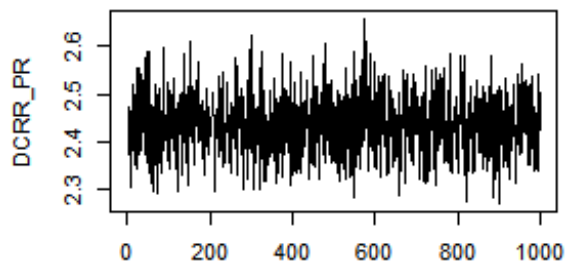

Histogram of DCRR_SE

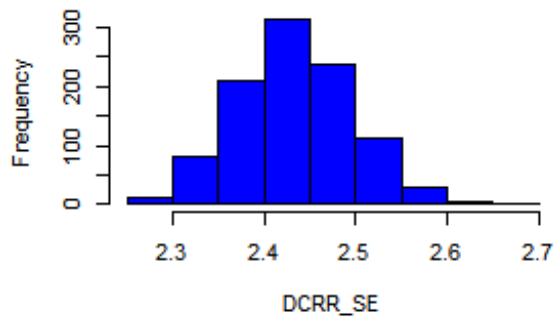

Histogram of DCRR_LINEx at $v=-1$

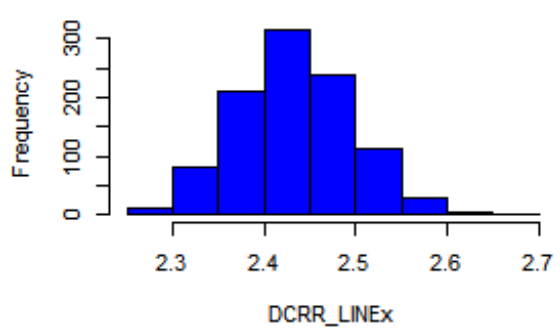

Histogram of DCRR_LINEx at $v=1$

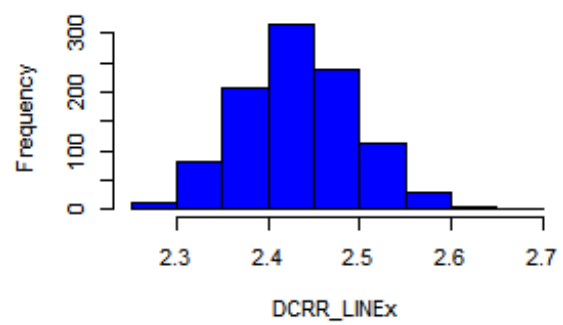

Histogram of DCRR_PR

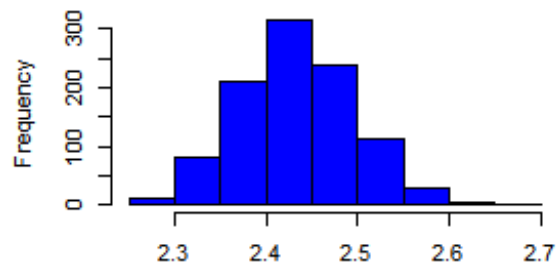

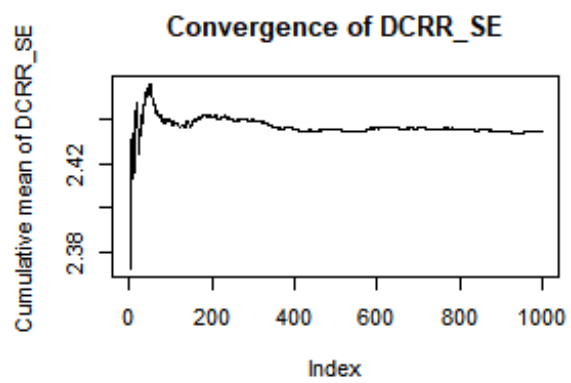
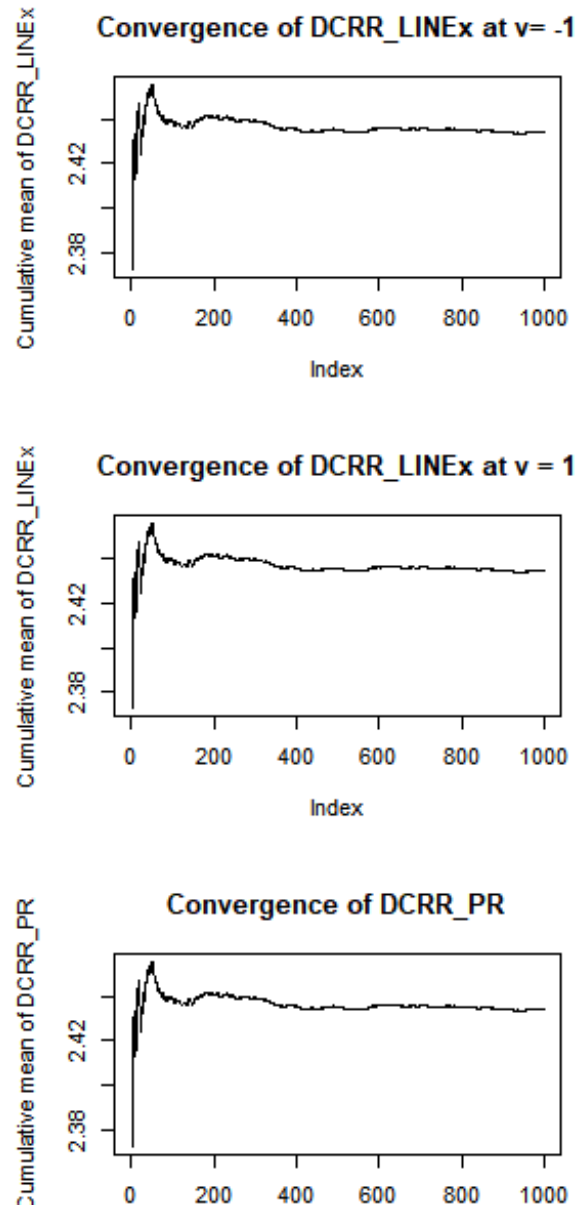

Figure 5. Example of convergence of MCMoC of estimates for $\gamma_{R}(\beta)$, at $t=0.5, \theta=0.8$, and $n=30$.

Table 7. DCRRéE Bayesian estimates at $t=0.5$ and 1.5 for elected loss functions.

\begin{tabular}{ccccc}
\hline$t$ & $\hat{\gamma}_{S E}(\boldsymbol{\beta})$ & $\hat{\gamma}_{\text {LINEx }}(\boldsymbol{\beta}),(\mathbf{v}=\mathbf{1})$ & $\hat{\gamma}_{\text {LINEx }}(\boldsymbol{\beta}),(\mathbf{v}=-\mathbf{1})$ & $\hat{\gamma}_{P R}(\boldsymbol{\beta})$ \\
\hline 0.5 & 3.711552 & 3.701186 & 3.703288 & 3.708402 \\
\hline 1.5 & 3.638074 & 3.634769 & 3.638159 & 3.635013 \\
\hline
\end{tabular}


Plot of DCRR_SE

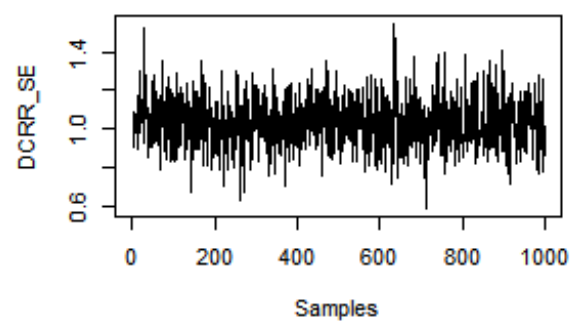

Plot of DCRR_LINEx at $v=-1$

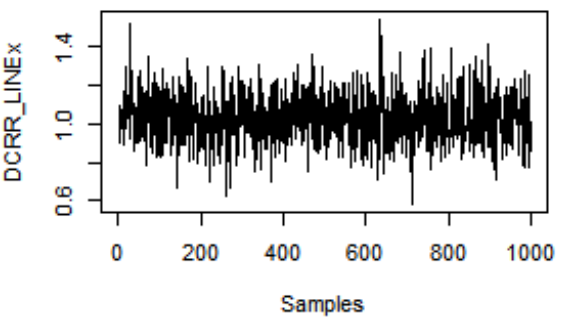

Plot of DCRR_LINEx at $v=1$

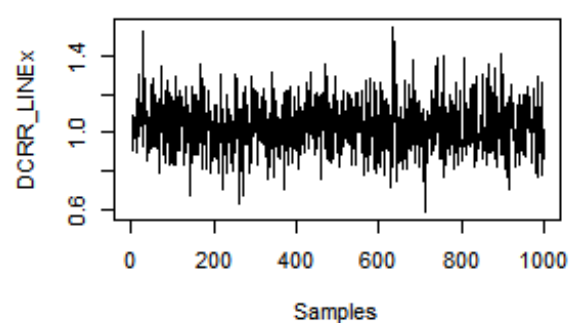

Plot of DCRR_PR

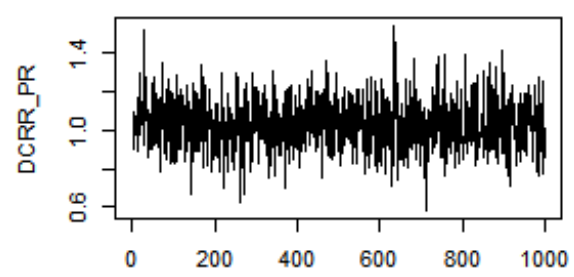

Histogram of DCRR_SE

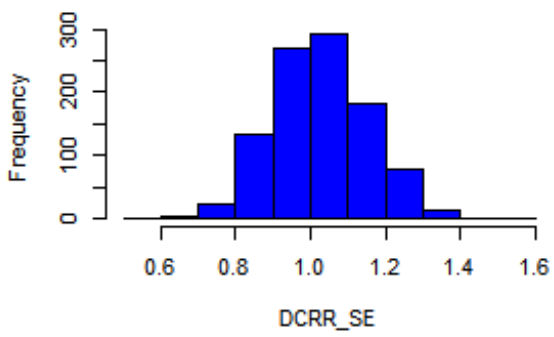

Histogram of DCRR_LINEx at $v=-1$
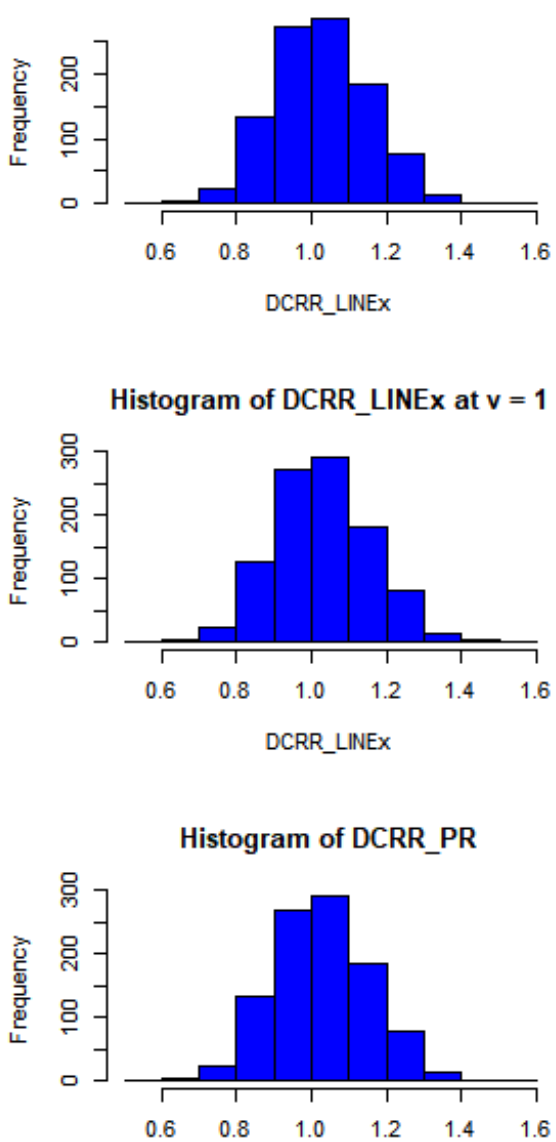
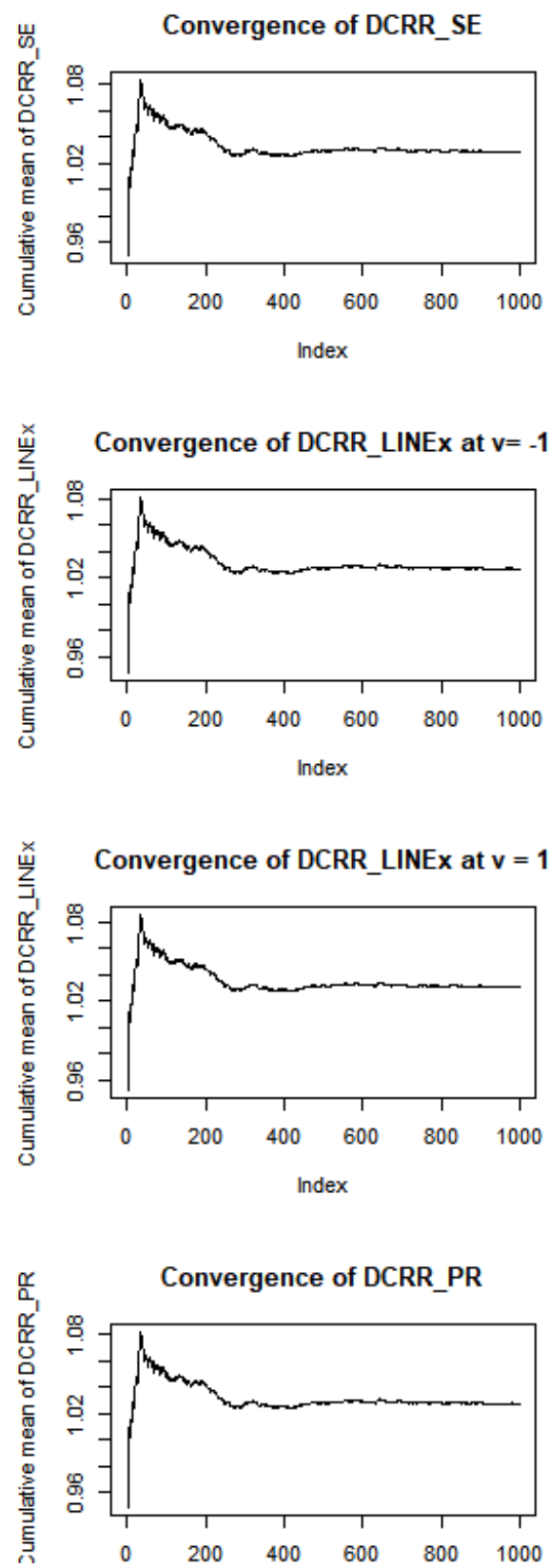

Figure 6. Example of convergence of MCMoC estimates for $\gamma_{R}(\beta)$, at $t=0.5, \theta=1.5$, and $n=100$.

As expected, the DCRRéE estimators for the proposed loss functions decrease with time, as seen in this example. 

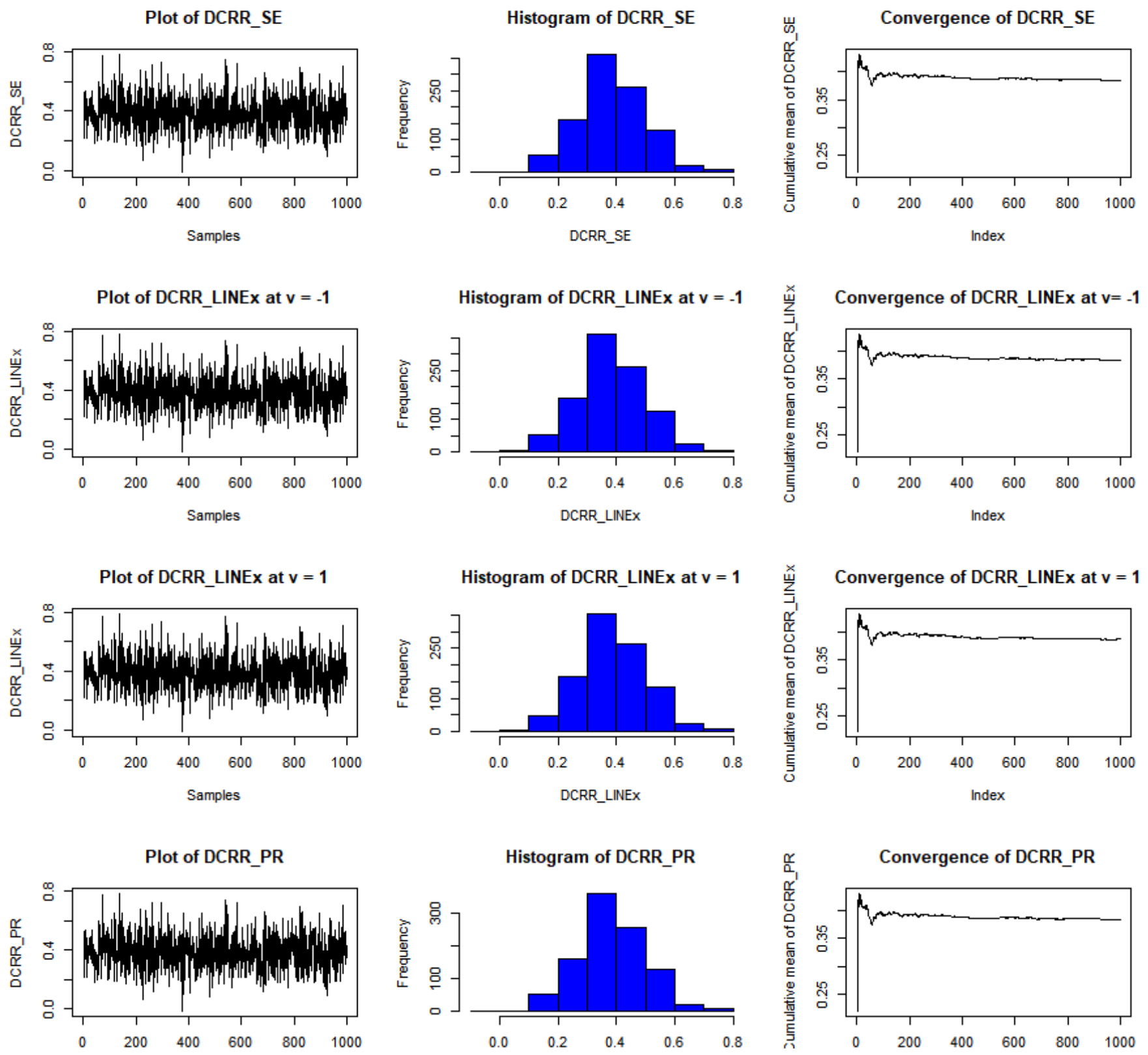

Figure 7. Example of convergence of MCMoC estimates for $\gamma_{R}(\beta)$, at $t=0.5, \theta=2.0$, and $n=50$.

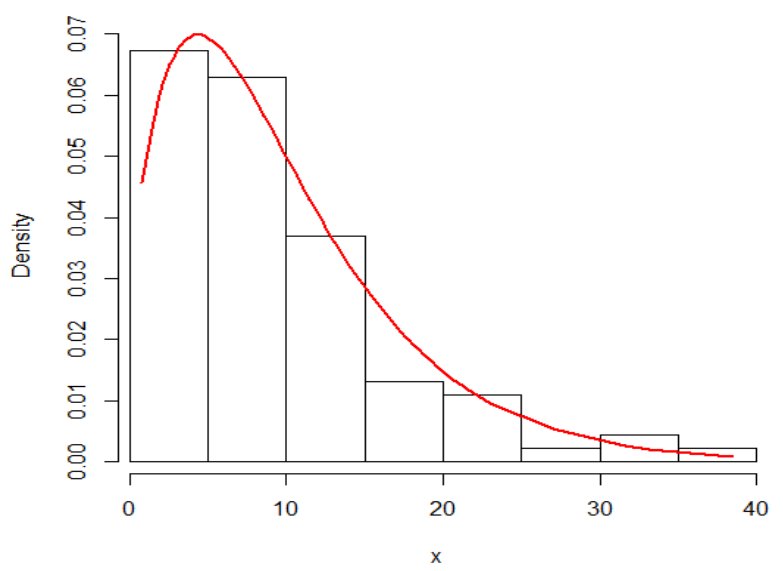

Figure 8. Fitted PDF plots of Lindley model for the data set. 


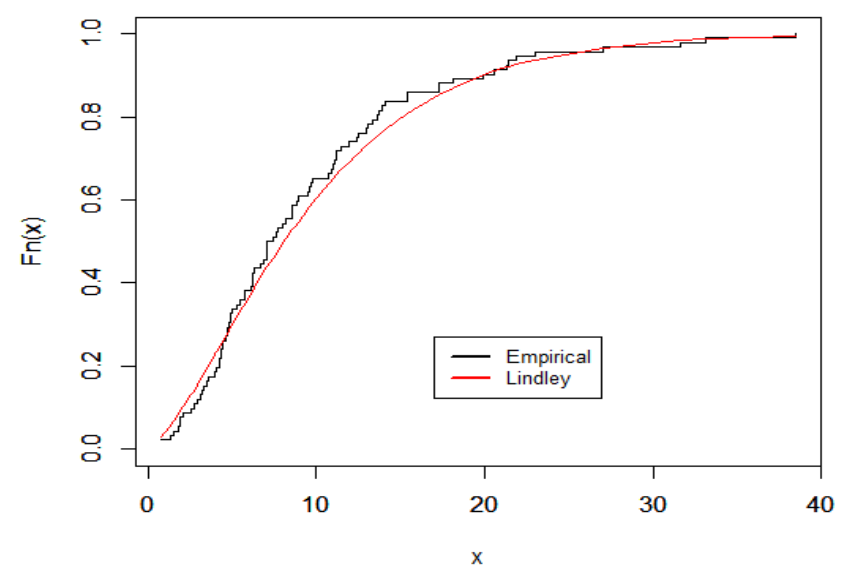

Figure 9. Fitted CDF plots of Lindley model for the data set.

\section{Concluding Remarks}

The Bayesian estimators of the DCRRéE for the Lindley distribution are investigated in this study. The Bayesian estimators of the DCRRéE for the Lindley model are thought to be produced by both symmetric and asymmetric loss functions. The MCMoC method is used to calculate the Bayesian estimator and the Bayesian credible intervals. The behavior of the DCRRéE estimators for the Lindley distribution is evaluated using some precision criteria. Real-world data and simulation concerns are addressed. Regarding the outcomes of the study, we conclude that for small actual values of the DCRRéE, the estimated risk and width of the Bayesian credible intervals of the DCRRéE estimates under the linear exponential loss function are often fewer than those based on the squared error and precautionary loss functions. At $t=0.5$, the width of the Bayesian credible intervals for the DCRRéE estimates via the linear exponential loss function is less than the others via the squared error and precautionary loss functions for a sample size of large values and large actual values of the DCRRéE. However, at $t=1.5$, the width of the Bayesian credible interval for the DCRRéE estimates via the precautionary loss function is smaller than the equivalent estimates via the squared error and linear exponential loss functions. For small DCRRéE values, the Bayesian estimates via the linear exponential loss function are preferable to other estimates under the squared error and precautionary loss functions. However, for a high true value of the DCRRéE, the Bayesian estimates under the precautionary loss function are preferable to the other estimates via the loss functions chosen.

Author Contributions: Methodology, A.M.A., A.A., A.S.H., A.N.Z. and M.E. Conceptualization, A.S.H.; Data curation, A.M.A.; Funding acquisition, A.M.A.; Methodology, A.A.; Project administration, M.E.; Software, A.N.Z.; Supervision, M.E. All authors have read and agreed to the published version of the manuscript.

Funding: The Deanship of Scientific Research (DSR), King Abdul-Aziz University, Jeddah, supported this work under grant no. (KEP-PhD-69-130-42). The authors, therefore, gratefully acknowledge the technical and financial support of the DSR.

Institutional Review Board Statement: Not applicable.

Informed Consent Statement: Not applicable.

Data Availability Statement: If you would like to obtain the numerical dataset used to conduct the study reported in the publication, please contact the appropriate author.

Acknowledgement:: We would like to express our gratitude to the two reviewers for their insightfuland useful suggestions on this manuscript, which significantly enhanced it. This project was funded by the Deanship of Scientific Research (DSR), King Abdul-Aziz University, Jeddah, supported this work under grant no. (KEP-PhD-69-130-42). The authors, therefore, gratefully acknowledge the technical and financial support of the DSR. 
Conflicts of Interest: The authors declare no conflict of interest.

\section{Acronyms \& Abbreviations}

$\begin{array}{ll}\text { BEs } & \text { Bayes estimates } \\ \text { CDF } & \text { Cumulative distribution function } \\ \text { DCR } & \text { dynamic cumulative residual } \\ \text { DCRRéE } & \text { dynamic cumulative residual Rényi entropy } \\ \text { ER } & \text { Estimated Risk } \\ \text { LINEx } & \text { Linear exponential loss function } \\ \text { L } & \text { Lower credible limit } \\ \text { MCMoC } & \text { Markov Chain Monte Carlo } \\ \text { MH } & \text { Metropolis-Hastings } \\ \text { PDF } & \text { Probability density function } \\ \text { PR } & \text { Precautionary loss function } \\ \text { RABs } & \text { Relative absolute biases } \\ \text { SE } & \text { Squared error loss function } \\ \text { SF } & \text { Survival function } \\ \text { U } & \text { Upper credible limit } \\ \text { WD } & \text { Width of credible intervals }\end{array}$

\section{References}

1. Rényi, A. On measures of Entropy and Information. In Proceeding of the Fourth Berkeley Symposium on Mathematical Statistics and Probability; University of California Press: Berkeley, CA, USA, 1961; Volume 1, pp. 547-561.

2. Renner, R.; Gisin, N.; Kraus, B. An information-theoretic security proof for quantum-key-distribution protocols. Phys. Rev. A 2005, 72, 1-18. [CrossRef]

3. Lévay, P.; Nagy, S.; Pipek, J. Elementary formula for entanglement entropies of fermionic systems. Phys. Rev. A 2005, 72, 1-8. [CrossRef]

4. Baratpour, S.; Ahmadi, J.; Arghami, N.R. Entropy properties of record statistics. Stat. Pap. 2007, 48, 197-213. [CrossRef]

5. Abo-Eleneen, Z.A. The entropy of progressively censored samples. Entropy 2011, 13, 437-449. [CrossRef]

6. Seo, J.I.; Lee, H.J.; Kan, S.B. Estimation for generalized half logistic distribution based on records. J. Korean Inf. Sci. Soc. 2012, 23, 1249-1257. [CrossRef]

7. Cho, Y.; Sun, H.; Lee, K. Estimating the Entropy of a Weibull Distribution under Generalized Progressive Hybrid Censoring Entropy 2015, 17, 102-122. [CrossRef]

8. Lee, K. Estimation of entropy of the inverse Weibull distribution under generalized progressive hybrid censored data. J. Korean Inf. Sci. Soc. 2017, 28, 659-668.

9. Chacko, M.; Asha, P.S. Estimation of entropy for generalized exponential distribution based on record values. J. Indian Soc. Probab. Stat. 2018, 19, 79-96. [CrossRef]

10. Al-Babtain, A.A.; Elbatal, I.; Chesneau, C.; Elgarhy, M. Estimation of different types of entropies for the Kumaraswamy distribution. PLoS ONE 2021, 16, e0249027. [CrossRef] [PubMed]

11. Hassan, A.S.; Zaky, A.N. Entropy Bayesian estimation for Lomax distribution based on record. Thail. Stat. 2021, 19, 96-115.

12. Hassan, A.S.; Zaky, A.N. Estimation of entropy for inverse Weibull distribution under multiple censored data. J. Taibah Univ. Sci. 2019, 13, 331-337. [CrossRef]

13. Rao, M.; Chen, Y.; Vemuri, B.C.; Wang, F. Cumulative residual entropy: A new measure of information. IEEE Trans. Inf. Theory 2004, 50, 1220-1228. [CrossRef]

14. Sunoj, S.M.; Linu, M.N. Dynamic cumulative rsidual Rényi's entropy. Statistics 2012, 46, 41-56. [CrossRef]

15. Kamari, O. On dynamic cumulative residual entropy of order statistics. J. Stat. Appl. Prob. 2016, 5, 515-519. [CrossRef]

16. Kundu, C.; Crescenzo, A.D.; Longobardi, M. On cumulative residual (past) inaccuracy for truncated random variables. Metrika 2016, 79, 335-356. [CrossRef]

17. Renjini, K.R.; Abdul-Sathar, E.I.; Rajesh, G. Bayes Estimation of Dynamic Cumulative Residual Entropy for Pareto Distribution Under Type-II Right Censored Data. Appl. Math. Model. 2016, 40, 8424-8434. [CrossRef]

18. Renjini, K.R.; Abdul-Sathar, E.I.; Rajesh, G. A study of the effect of loss functions on the Bayes estimates of dynamic cumulative residual entropy for Pareto distribution under upper record values. J. Stat. Comput. Simul. 2016, 86, 324-339. [CrossRef]

19. Renjini, K.R.; Abdul-Sathar, E.I.; Rajesh, G. Bayesian estimation of dynamic cumulative residual entropy for classical Pareto distribution. Am. J. Math. 2018, 37, 1-13. [CrossRef]

20. Ahmadini, A.A.H.; Hassan, A.S.; Zaki, A.N.; Alshqaq, S.S. Bayesian Inference of Dynamic Cumulative Residual Entropy from Parto II distribution with Application to COVID-19. AIM Math. 2020, 6, 2196-2216. [CrossRef]

21. Al-Babtain, A.A.; Hassan, A.S.; Zaky, A.N.; Elbatal, I.; Elgarhy, M. Dynamic cumulative residual Rényi entropy for Lomax distribution: Bayesian and non-Bayesian methods. AIM Math. 2021, 6, 3889-3914. [CrossRef] 
22. Lindley, D.V. Fiducial distributions and Bayes' theorem. J. R. Stat. Soc. B 1958, 20, 102-107. [CrossRef]

23. Ghitany, M.E.; Atieh, B.; Nadarajah, S. Lindley distribution and its application. Math. Comput. Simul. 2008, 78, 493-506. [CrossRef]

24. Ghitany, M.E.; Alqallaf, F.; Al-Mutairi, D.K.; Husain, H.A. A two-parameter Lindley distribution and its applications to survival data. Math. Comput. Simul. 2011, 81, 1190-1201. [CrossRef]

25. Ali, S.; Aslam, M.; Kazmi, S.M.A. A study of the effect of the loss function on Bayes Estimate, posterior risk and hazard function for Lindley distribution. Appl. Math. Model. 2013, 37, 6068-6078. [CrossRef]

26. Al-Mutairi, D.K.; Ghitany, M.E.; Kundu, D. Inferences on stress-strength reliability from Lindley distributions. Commun. Stat. Theory Methods 2013, 42, 1443-1463. [CrossRef]

27. Krishna, H.; Kumar, K. Reliability estimation in Lindley distribution with progressively type II right censored sample. Math. Comput. Simul. 2011, 82, 281-294. [CrossRef]

28. Sharma, V.K.; Singh, S.K.; Singh, U.; Ul-Farhat, K. Bayesian estimation on interval censored Lindley distribution using Lindley's approximation. Int. J. Syst. Assur. Eng. Manag. 2016, 8, 799-810. [CrossRef]

29. Maiti, S.S.; Mukherjee, I. On estimation of the PDF and CDF of the Lindley distribution. Commun. Stat. Simul. Comput. 2018, 47, 1370-1381. [CrossRef]

30. Hafez, E.H.; Riad, F.H.; Mubarak, S.A.M.; Mohamed, M.S. Study on Lindley distribution accelerated life tests: Application and numerical simulation. Symmetry 2020, 12, 2080. [CrossRef]

31. Chen, M.H.; Shao, Q.M. Monte Carlo Estimation of Bayesian Credible and HPD Intervals. J. Comput. Graph. Stat. 1999, 8, 69-92.

32. Metropolis, N.; Rosenbluth, A.W.; Rosenbluth, M.N.; Teller, A.H.; Teller, E. Equations of state calculations by fast computing machines. J. Chem. Phys. 1953, 21, 1087-1091. [CrossRef] 